\title{
reviscafuences
}

ISSN: $1575-7072$ | $6.155 \mathrm{~N}: 2172.7775$

Páginas: $138-149$

Recibido: 2020-09-25

Revisado: 2020-11-13

Aceptado: 2021-03-29

Preprint: 2021-05-10

Publicación Final: 2021-05-15

www.revistascientificas.us.es/index.php/fuentes/index

DOI: https://doi.org/10.12795/revistafuentes.2021.12753

\section{El impacto de las tecnologías en el ocio de la primera infancia}

\section{The impact of technologies on early childhood leisure}

\author{
iD Laura Díaz-Herrera \\ Universidad de Cantabria (España) \\ iD Irina Salcines-Talledo \\ Universidad de Cantabria (España) \\ iD Natalia González-Fernández \\ Universidad de Cantabria (España)
}

\section{Resumen}

En los últimos años, se advierte un incremento considerable en la importancia que toman las Tecnologías de la Información y de la Comunicación (TIC) dentro del tiempo de ocio de la primera infancia. En este contexto, es fundamental atender al papel de las familias a la hora de regular su uso, convirtiéndose así en las responsables últimas de orientar a los menores para prevenir sus riesgos y beneficiarse de sus potencialidades. El objetivo de esta investigación es reconocer el impacto de las TIC en las actividades de ocio durante el tiempo libre de los menores de 3-6 años de una localidad de Cantabria. Para ello, se emplea un cuestionario diseñado ad hoc, el cual ha sido aplicado a 149 familias seleccionadas mediante muestreo no probabilístico de tipo incidental. Los resultados señalan que las actividades que recurren al soporte tecnológico se configuran como un pilar fundamental del tiempo de ocio de los más pequeños, constituyendo la diversión y el aprendizaje recreativo las principales intenciones que se plantean las familias a la hora de exponer a los niños a las TIC. Las conclusiones apuntan a la necesidad de concienciar a los progenitores de su importancia como modeladores del uso tecnológico desarrollado por sus hijos, dotándoles de algunas claves para emprender esta labor de manera eficaz.

\section{Abstract}

In recent years, a great increase in the importance of Information and Communication Technology (ICT) during early childhood leisure time has been noted. In this context, it is necessary to pay attention to the role of families in order to regulate their use, becoming the main responsible for guiding children to prevent their risks and benefit from their potential This project aims to recognize the impact of ICT in leisure activities during 3-6 years old children's free time in a town of Cantabria. According to this aim, an ad hoc questionnaire is used to collect the information provided by 149 families selected through a non-probabilistic sampling of an incidental type. The results show that activities which use the technological support are configured as a fundamental pillar of children's leisure time, being enjoyment and recreational learning the main purposes considered by families when exposing their children to ICT. The conclusions point to the need of making parents aware of their importance as modelers of the technological use developed by their children, providing them with some keys to undertake this task effectively.

\section{Palabras clave}

ocio, primera infancia, familias, tiempo libre, diversión, aprendizaje recreativo, riesgos.

\section{Keywords}

ICT, leisure, early childhood, families, free time, enjoyment, recreational learning, risks. 


\section{Introducción}

Hoy en día, nos situamos en el contexto de la denominada "cuarta gran revolución digital" o "revolución 4.0", basada en el uso de la tecnología digital, la inteligencia artificial, la globalización y la hiperconectividad (LeónLlorente, 2020). Así, nos encontramos ante una generación de niños con acceso a las Tecnologías de la Información y de la Comunicación (TIC) desde su nacimiento, a la cual Prensky (2001) acuñó como "nativos digitales". Más adelante, Turk (2018) alude a esta generación con el término "Generación Alfa", acuñado por Mark McCrindle para designar a los menores nacidos a partir del año 2010. De este modo, se explica cómo -a pesar de tratarse de una generación colindante en el tiempo con los denominados "Millennials" o "Generación Z"- los miembros de la Generación Alfa varían significativamente respecto a los anteriores en relación con sus intereses y motivaciones, lo cual responde fundamentalmente a los cambios impuestos por los avances tecnológicos. En esta línea, hay constancia de que el tiempo de uso de las TIC por parte de los más pequeños ha aumentado exponencialmente durante los últimos años, así como lo ha hecho la diversidad y la especialización de los dispositivos a la vez que se ha democratizado su uso (CEPAL \& UNICEF, 2016; Soto et al., 2018). Un claro síntoma de lo anterior es recogido por el Instituto Nacional de Estadística (INE) (2019), dejando latente cómo actualmente un 92.9\% de los menores tienen acceso habitual a Internet.

En lo que respecta a la edad de inicio en el uso de las TIC, hay constancia de que los niños de 1-3 años ya tienen acceso habitual a los dispositivos de sus familias, si bien la edad que los progenitores consideran razonable para la tenencia de dispositivos propios oscila en torno a los 10-13 años (Cánovas et al., 2014; Chaudron et al., 2018; GAD3, 2018; Roca, 2015; Sivrikova et al., 2020). No obstante, la frecuencia y la intensidad de empleo de las mismas tiende a aumentar con la edad, destacando un auge importante alrededor de los 13 años (INE, 2019; Labrador et al., 2015). Inmersos en su uso, en las últimas investigaciones se aprecia una tendencia a que sean los dispositivos móviles -más concretamente, tablet y smartphone- los utilizados con mayor frecuencia por los menores (Chaudron et al., 2018; Garmendia et al., 2016; Instituto Nacional de Tecnologías Educativas y de Formación del Profesorado [INTEF], 2016; Fernández, 2017; Martínez et al., 2019; Pérez, 2018). Respecto a su empleo preferente, los niños de más cortas edades parecen interesarse en el juego y los dibujos animados, mientras que -con el paso del tiempo- comienzan a mostrar interés por la producción de contenido audiovisual (Cánovas et al., 2014; Carrillo, 2015; Chaudron et al., 2018; INTEF, 2016; Soto et al., 2018). Sin embargo, cabe señalar que -a pesar del incremento en el empleo de los dispositivos móviles- la televisión continúa siendo utilizada con gran asiduidad por parte de los más pequeños, con tendencia a aumentar cuando la edad de los niños es menor (Chaudron et al., 2018; Feijóo, 2015; GAD3, 2018).

Atendiendo al incremento en el uso de las TIC por parte de los menores, resulta inherente a ello el hecho de que estas tecnologías ocupen cada vez un espacio mayor dentro del tiempo de ocio de la infancia. Así, más allá del empleo de los dispositivos de sus familias, actualmente las TIC se posicionan como uno de los regalos más demandados por los niños, aumentando exponencialmente su uso en detrimento de otras actividades como el juego tradicional, el deporte o la literatura (Cánovas et al., 2014; Feijóo, 2015; Paniagua, 2018). Situados en este contexto, cabe replantearse cuáles son las virtudes y los riesgos que puede suponer el uso de las TIC para los más pequeños. De esta forma, mientras que los beneficios que ofrecen son indudables -facilitan comunicaciones a distancia y favorecen oportunidades relacionadas con la educación, la recreación y la participación social, rescatando la diversidad y la pluralidad- un empleo inapropiado de las mismas puede dar lugar a problemáticas como la adicción o la posibilidad de que otras propuestas de ocio tomen un segundo plano (Cruz et al., 2019; Labrador et al., 2015; López-Castro et al., 2019; Muñoz-Borja et al., 2020; Roca, 2015).

Comenzando por el posible impacto positivo, la oportunidad de que los menores se adentren en el mundo de las TIC a edades tempranas conlleva un doble beneficio: tienen una alta receptividad hacia lo que se les enseña acerca del uso de las mismas, a la vez que aprenden de manera lúdica. Esto permite plantear el primer acercamiento a las TIC como una oportunidad educativa para las familias, lo cual toma más importancia aún en la etapa 0-6, que configura el contacto inicial de los menores con el mundo tecnológico (Cánovas et al., 2014; Gjelaj et al., 2020). Asimismo, otro de los grandes beneficios es el apoyo que ofrecen para las personas con discapacidad, pudiendo llegar a convertirse -según UNICEF (2017)- en "la diferencia entre la exclusión social y la igualdad de oportunidades" (p.17). En este sentido, cabe recuperar algunas experiencias dirigidas a menores diagnosticados con Trastorno del Espectro Autista (TEA) o con discapacidad auditiva, así como a niños con trastornos específicos del habla y con problemas respiratorios, a los cuales se les ofrecen aplicaciones y programas gratuitos con el objetivo de facilitar su comunicación, su aprendizaje, su recreación e incluso su rehabilitación en el último de los casos (Alegre \& Villar, 2019; Espinoza-Santacruz \& Flores-Urgiles, 2019; Roca, 2015). Finalmente, resulta pertinente mencionar de qué forma los juegos educativos estandarizados y los videojuegos de carácter formal e informal comportan mejoras en áreas tan diversas como la conciencia fonológica, la comprensión lectora, la adquisición de vocabulario, la atención, la resolución de problemas, el pensamiento lógico-matemático, el desarrollo del lenguaje, la toma de decisiones, la responsabilidad y la 
autonomía, la adquisición de una segunda lengua o la velocidad de los actos reflejos, entre otras (Álvarez \& Alejaldre, 2019; López-Cirugeda \& López-Campillo, 2016; Roca, 2015; Rojo \& Dudu, 2017; Serna-Rodrigo, 2020; Takacs et al., 2015).

Por el contrario, y sin negar los múltiples beneficios de las TIC, también resulta preciso conocer los factores de riesgo que pueden vincularse al empleo de las mismas. En este sentido, se hablaría de cómo los juegos digitales tienden a fomentar el individualismo en detrimento de las relaciones sociales, así como existe el riesgo de impacto de valores negativos que, en ocasiones, transmiten los mismos (Fernández, 2017; Paniagua, 2018). Ante esta problemática, lo verdaderamente importante es que las familias conozcan cómo son los juegos que frecuentan los menores, así como que revisen el número de horas en las que sus hijos están expuestos a ellos (Academia Americana de Pediatría, 2016; Organización Mundial de la Salud [OMS], 2019; Roca, 2015; UNICEF, 2017). No obstante, es fundamental que no se limiten a controlar el empleo de los juegos, sino que sean capaces de plantear otras alternativas para el desarrollo de un ocio saludable por parte de los más pequeños.

De igual forma, dentro del estudio desarrollado por GAD3 (2018) se menciona el concepto de "niños Amazon", con el cual se alude a la generación de niños familiarizados con la inmediatez de respuesta a sus necesidades. Es de esta forma como está desapareciendo el valor de la espera. Según las palabras de L'Ecuyer (2017), sobreestimular a los menores -ofreciéndoles respuestas inmediatas e incluso anticipándose a sus necesidadesanula su capacidad de ilusionarse y de asombrarse, esto es, les arrebata la posibilidad de motivarse por sí mismos. En este sentido, e inmersos en una sociedad en la que predomina la fugacidad y la inminencia, resulta conveniente que los padres sean capaces de ralentizar el ritmo de vida de los niños. Esto no implica dejar de responder a sus necesidades, pero sí valorar el grado de inmediatez que pueden requerir las mismas. A partir de ahí, es determinante que los progenitores sean capaces de aportar la tranquilidad necesaria para que los menores puedan huir de la celeridad y comiencen a disfrutar plenamente de las actividades, sin prisas ni apremio por finalizarlas.

Por otra parte, el hecho de permanecer un elevado número de horas frente a las pantallas se asocia con un mayor consumo de alimentos poco saludables, así como con una menor frecuencia de realización de ejercicio físico, síntomas directos de la obesidad infantil (Díaz \& Aladro, 2016; Paniagua, 2018). Para prevenir estos trastornos, la actividad física es considerada garante de mejora de la calidad de vida de los más pequeños, siendo recomendable que los menores a partir del primer año se mantengan físicamente activos durante al menos 180 minutos al día, con un incremento consecuente en el tiempo a medida que aumenta su edad, a la vez que se produzca una restricción necesaria en el tiempo de uso de las TIC (Álvarez-Pitti et al., 2020; OMS, 2019; Roca, 2015).

Finalmente, entre las causas que pueden conllevar un empleo excesivo de las TIC, cabe destacar una que resulta en muchos casos determinante: la exposición de los niños a las TIC por comodidad. Así, en la investigación desarrollada por Chaudron et al. (2018) se observa cómo las familias recurren a las TIC cuando necesitan "tiempo para ellos" o en situaciones en las que pretenden mantener a los niños tranquilos, sobre todo fuera del hogar. Lamentablemente, su empleo con estos fines no materializa la posibilidad que se mencionaba previamente de educar a los niños en el manejo eficiente de las TIC, sino que se sitúa como un mero recurso de entretenimiento cómodo y sencillo para los padres (Cánovas et al., 2014).

Las problemáticas anteriores -entre otras- derivan en lo que se entiende como un uso abusivo de las TIC, localizado en el punto en el que el empleo de las mismas actúa en detrimento de sus obligaciones, de otras aficiones y de sus relaciones sociales, llegando a comprometer la vida cotidiana de los menores (Labrador et al., 2015; Paniagua, 2018). Con la intención de minimizar este riesgo, es importante sensibilizar a las familias, haciéndoles conscientes de la necesidad de establecer límites claros y explícitos en el uso de las TIC, así como favorecer estrategias y herramientas que les permitan sentirse más competentes en lo relativo a su comprensión y a su empleo para poder aportar un buen modelo de uso digital a los menores (Fernández, 2017; Ramírez-García et al., 2020; Roca, 2015; Soto et al., 2018; UNICEF, 2017). En relación a la regulación, la mayor parte de las familias se muestra a favor del establecimiento de medidas de control en el empleo de las TIC, entendiendo la seguridad y la privacidad de los niños de forma prioritaria (GAD3, 2018; Sivrikova et al., 2020). Sin embargo, en lo que respecta a las competencias mencionadas previamente, es habitual que los adultos consideren que sus hijos -como "nativos digitales"- son más eficientes en el uso de las TIC que ellos mismos (UNICEF, 2014; UNICEF, 2018). Esta consideración es errónea, ya que su capacidad de seleccionar información y de prevenir los riesgos de privacidad y de veracidad es muy limitada, siendo este el punto en el que las familias -y también las escuelas- han de actuar (Cánovas et al., 2014; Quiroga et al., 2019; Roca, 2015). Para afrontar este desafío, es necesario ofrecer a las familias -especialmente a aquellas cuyas posibilidades de acceso a recursos educativos, como libros o revistas especializadas, son más limitadas- propuestas formativas acerca del uso de las TIC en el hogar, pudiendo tomar la figura del orientador en los centros educativos una posición muy interesante al respecto (Ramírez-García et al., 2020). 
Atendiendo a lo expuesto, la presente investigación se plantea el objetivo de reconocer el impacto de las TIC en el ocio de los menores de 3-6 años de una localidad de Cantabria. Para ello, se ha diseñado un cuestionario original que ha permitido recabar la información aportada por 149 familias con hijos en diferentes centros educativos de la localidad.

\section{Metodología}

La presente investigación ha sido estructurada mediante un enfoque metodológico cuantitativo descriptivo y un diseño no experimental, puesto que se han analizado y descrito las variables que constituyen el fenómeno a investigar en su situación natural, recurriendo al empleo de un cuestionario como técnica de recogida de información (Albert, 2007; Hernández et al., 2006; Hernández et al., 2007).

\subsection{Muestra}

Para la selección de la muestra, se ha seguido un muestreo no probabilístico de tipo incidental (Sáez, 2017), es decir, se ha trabajado con una muestra elegida intencionalmente por la oportunidad de acceso y cercanía a la misma.

En la investigación han participado un total de 149 familias con niños de entre 3 y 6 años de una localidad de Cantabria, cuyas características se recogen en la Tabla 1.

\section{Tabla 1}

Resumen de frecuencias y porcentajes de las familias participantes

\begin{tabular}{|l|l|l|l|}
\hline \multirow{5}{*}{ Edad } & De 20 a 30 años & $\mathbf{N}$ & $\%$ \\
& De 31 a 40 años & 96 & 6.04 \\
Género & Más de 40 años & 44 & 2.43 \\
& Masculino & 17 & 11.53 \\
Nivel de estudios & Femenino & 130 & 87.25 \\
& Otro & 2 & 1.34 \\
& Superiores & 86 & 57.72 \\
Situación laboral & FP & 38 & 25.50 \\
& Secundarios & 21 & 14.09 \\
& Primarios y sin estudios & 4 & 2.68 \\
& Empleado & 119 & 79.87 \\
& Desempleado & 24 & 16.11 \\
Estructura familiar & Jubilado / Pensionista & 2 & 1.34 \\
& Estudiante & 4 & 2.64 \\
& Nuclear & 133 & 89.26 \\
& Monoparental & 9 & 6.04 \\
& Homoparental & 1 & .67 \\
& Adoptiva & 1 & .67 \\
& Reconstituida & 3 & 2.01 \\
& Extensa & 2 & 1.34 \\
\hline
\end{tabular}

En los datos reflejados por las familias se observa una muestra feminizada, constituyendo las madres integrantes, en su mayoría, de estructuras nucleares- la principal fuente de información de la que se dispone. Por su parte, más de la mitad de la muestra tiene edades comprendidas entre 31 y 40 años, así como se percibe la primacía de estudios superiores. En cuanto a la situación laboral, más de tres cuartas partes de los participantes son actualmente empleados. Finalmente, los ingresos económicos netos mensuales oscilan habitualmente entre los 2101 y los 2800 euros, con una mayor diversidad de respuestas en esta variable.

Seguidamente, los datos relativos a la información que aportan los progenitores sobre sus hijos quedan reflejados en la Tabla 2. 
Tabla 2

Datos identificativos de los menores

\begin{tabular}{|c|c|c|c|c|c|c|c|c|}
\hline $\begin{array}{l}\text { VARIABLE } \\
\text { Hijo } 1 \\
\text { Sexo } \\
\text { Niño } \\
\text { Niña } \\
\text { Edad } \\
3 \text { años } \\
4 \text { años } \\
5 \text { años } \\
6 \text { años }\end{array}$ & $\begin{array}{l}\mathbf{N} \\
148\end{array}$ & $\begin{array}{l}59.46 \\
40.54 \\
20.41 \\
21.09 \\
25.85 \\
32.65\end{array}$ & $\begin{array}{l}\text { VARIABLE } \\
\text { Hijo } 2 \\
\text { Sexo } \\
\text { Niño } \\
\text { Niña } \\
\text { Edad } \\
3 \text { años } \\
4 \text { años } \\
5 \text { años } \\
6 \text { años }\end{array}$ & $\begin{array}{l}\mathbf{N} \\
34\end{array}$ & $\begin{array}{l}47.06 \\
52.94 \\
64.71 \\
20.59 \\
8.82 \\
5.88\end{array}$ & $\begin{array}{l}\text { VARIABLE } \\
\text { Hijo } 3 \\
\text { Sexo } \\
\text { Niña } \\
\text { Edad }\end{array}$ & 1 & 100.00 \\
\hline
\end{tabular}

Se percibe cómo 34 de las 148 familias participantes tienen un segundo hijo con edad entre 3 y 6 años, mientras que únicamente 1 tiene un tercer hijo que cumpla dicha premisa. Así, se trabaja con datos de 183 menores.

En cuanto al sexo, se observa cómo en el caso del primer hijo, el porcentaje de niños es superior al de niñas, tendencia que se invierte con el segundo. Finalmente, el único caso relativo al tercer hijo es una niña.

En relación con la edad, en el caso del primer hijo el porcentaje de niños aumenta a la vez que lo hace la edad del menor, teniendo 3 años los más pequeños y 6 años los más mayores, con una media de edad de ( $\mathrm{M}=$ 4.71 , d.t. $=1.13$ ). Por su parte, la tendencia se desarrolla a la inversa en el caso del segundo hijo, con un valor medio de edad de $(M=3.56$, d.t. $=.89)$.

\subsection{Instrumento}

Se presenta un cuestionario sobre la promoción del uso de las TIC por las familias en el ocio de los menores, el cual ha sido diseñado específicamente para la investigación. Dicho instrumento está formado por 31 preguntas cerradas y de tipo Likert (escala 1 a 5) distribuidas en seis bloques.

Para su validación, se ha consultado a tres jueces expertos en la temática sobre la pertinencia y la idoneidad de la primera versión del instrumento a través de una plantilla de validación en la que se pregunta sobre el contenido, el orden, la claridad y la extensión del cuestionario, así como su adecuación a los destinatarios. A partir de ahí, se han analizado las sugerencias aportadas por los profesionales expertos y se ha confeccionado la versión final del instrumento.

Por su parte, con la intención conocer la validez interna del cuestionario se ha realizado un análisis de fiabilidad mediante la prueba Alfa de Cronbach, evaluando el cuestionario global tras eliminar los ítems identificativos. El resultado certifica una fiabilidad adecuada de la herramienta, con un valor $\alpha$ de .75.

\subsection{Análisis de datos}

El análisis de datos se ha realizado con el programa estadístico SPSS v.22. En este sentido, se han desarrollado análisis descriptivos y de frecuencias, así como estadística inferencial no paramétrica. Una vez realizada la prueba Kolmogorov-Smirnov, y comprobado que en la totalidad de los casos $(p=.000)$, se descarta que la muestra cumpla el criterio de normalidad.

\section{Resultados}

\subsection{Tiempo de uso de las TIC}

A continuación, la Tabla 3 recoge -expresados en porcentaje- los valores relativos al tiempo de uso de las TIC por parte de los menores. 
Tabla 3

Tiempo de uso de las TIC por parte de los menores

\begin{tabular}{|c|c|c|c|c|c|}
\hline ÍTEM & Ninguna & Menos de & $\begin{array}{l}\text { De una a } \\
\text { tres }\end{array}$ & $\begin{array}{l}\text { De tres a } \\
\text { seis }\end{array}$ & Más de seis \\
\hline $\begin{array}{l}\text { B.1. Número de horas diarias de } \\
\text { uso de las TIC en solitario entre } \\
\text { semana. }\end{array}$ & 12.80 & 47.00 & 28.90 & 6.70 & 4.70 \\
\hline $\begin{array}{l}\text { B.2. Número de horas diarias de } \\
\text { uso de las TIC en solitario durante } \\
\text { los fines de semana. }\end{array}$ & 12.80 & 21.50 & 52.30 & 10.70 & 2.70 \\
\hline $\begin{array}{l}\text { B.3. Número de horas diarias de } \\
\text { uso de las TIC en familia entre } \\
\text { semana. }\end{array}$ & 20.80 & 55.00 & 18.10 & 3.40 & 2.70 \\
\hline $\begin{array}{l}\text { B.4. Número de horas diarias de } \\
\text { uso de las TIC en familia durante los } \\
\text { fines de semana. }\end{array}$ & 9.40 & 40.90 & 41.60 & 6.00 & 2.00 \\
\hline
\end{tabular}

De acuerdo con los datos, se observa que los valores más elevados de horas de uso de las TIC por parte de los menores se concentran en los fines de semana, ya sea en solitario $(M=2.69$, d.t. $=.92)$ o en compañía de los progenitores $(\mathrm{M}=2.50$, d.t. $=.83)$. Por el contrario, la puntuación inferior se localiza en el tiempo de uso de las TIC compartido con la familia entre semana $(\mathrm{M}=2.12$, d.t. $=.87)$.

A partir de lo anterior, se ha considerado pertinente agrupar los cuatro ítems en una misma variable, denominada "Tiempo de uso de las TIC" (Tabla 4).

Tabla 4

Descriptivos de la variable "Tiempo de uso de las TIC"

\begin{tabular}{|l|c|c|c|c|c|}
\hline VARIABLE & $\mathbf{N}$ & Mínimo & Máximo & Media & Desviación típica \\
$\begin{array}{l}\text { Tiempo de uso } \\
\text { de las TIC }\end{array}$ & 149 & 1.00 & 5.00 & 2.44 & .73 \\
\hline
\end{tabular}

Se interpreta, así, que la puntuación media de la variable es inferior al punto medio de la escala, 3 (escala de 1 a 5, siendo 1: Ninguna, 2: Menos de una, 3: De una a tres, 4: De tres a seis y 5: Más de seis), quedando reflejado cómo el número medio de horas diarias que dedican los menores al uso de las TIC -independientemente de los demás condicionantes- oscila alrededor de 1 hora.

\subsubsection{Tiempo de uso de las TIC y edad de los menores}

Considerando el tiempo de uso de las TIC anteriormente expuesto, se pretende comprobar cómo influye la variable independiente "Edad" en las puntuaciones del mismo. Así, en la Tabla 5 aparecen reflejados los datos descriptivos que se han extraído.

Tabla 5

Tiempo de uso de las TIC en función de la edad

\begin{tabular}{|l|c|c|c|}
\hline & $\mathbf{N}$ & Media & Desviación típica \\
$\mathbf{3}$ años & 30 & 2.32 & .78 \\
$\mathbf{4}$ años & 31 & 2.44 & .61 \\
$\mathbf{5}$ años & 38 & 2.41 & .57 \\
$\mathbf{6}$ años & 48 & 2.53 & .87 \\
\hline
\end{tabular}

De acuerdo con los datos anteriores, cabe destacar cómo la puntuación más elevada se encuentra en "6 años", lo que muestra una tendencia a que a mayor edad, mayor sea el tiempo de uso de las TIC. A partir de ahí, con la intención de comprobar si existen diferencias significativas en alguno de los ítems, se ha llevado a cabo la prueba Kruskal-Wallis. Conocidos los resultados, se puede afirmar que solo existen diferencias significativas en lo relativo a "Número de horas diarias de uso de las TIC durante los fines de semana" $\left(\chi^{2}=9.096, \mathrm{gl}=3\right.$, $\mathrm{p}=.028)$.

Por último, con la finalidad de comprobar entre qué edades existe una diferencia significativa, se ha desarrollado la prueba $U$ de Mann-Whitney (Tabla 6). Los resultados constatan que existen diferencias significativas en el número de horas de uso de las TIC durante los fines de semana entre los niños de 3 años 
y los niños de 6 años, siendo los segundos quienes utilizan las TIC un número de horas significativamente superior a los primeros.

Tabla 6

Prueba $U$ de Mann-Whitney en función de la edad de los menores

\begin{tabular}{l|c|c|c}
\hline Ítem & Grupos de edad & U de Mann-Whitney & Significación asintótica \\
& 3 años -4 años & 365.500 & .127 \\
Número de horas diarias & 3 años - 5 años & 444.500 & .092 \\
de uso de las TIC durante & 3 años - 6 años & 467.500 & .006 \\
los fines de semana & 4 años - 5 años -6 años & 582.000 & .923 \\
& 5 años - 6 años & 609.500 & .140 \\
\hline
\end{tabular}

\subsection{Edad a la que los menores entran en contacto con las TIC}

Analizando las respuestas a los ítems relativos a la edad en la que los menores entran en contacto con los diferentes dispositivos tecnológicos, los datos recogidos quedan reflejados en la Figura 1.

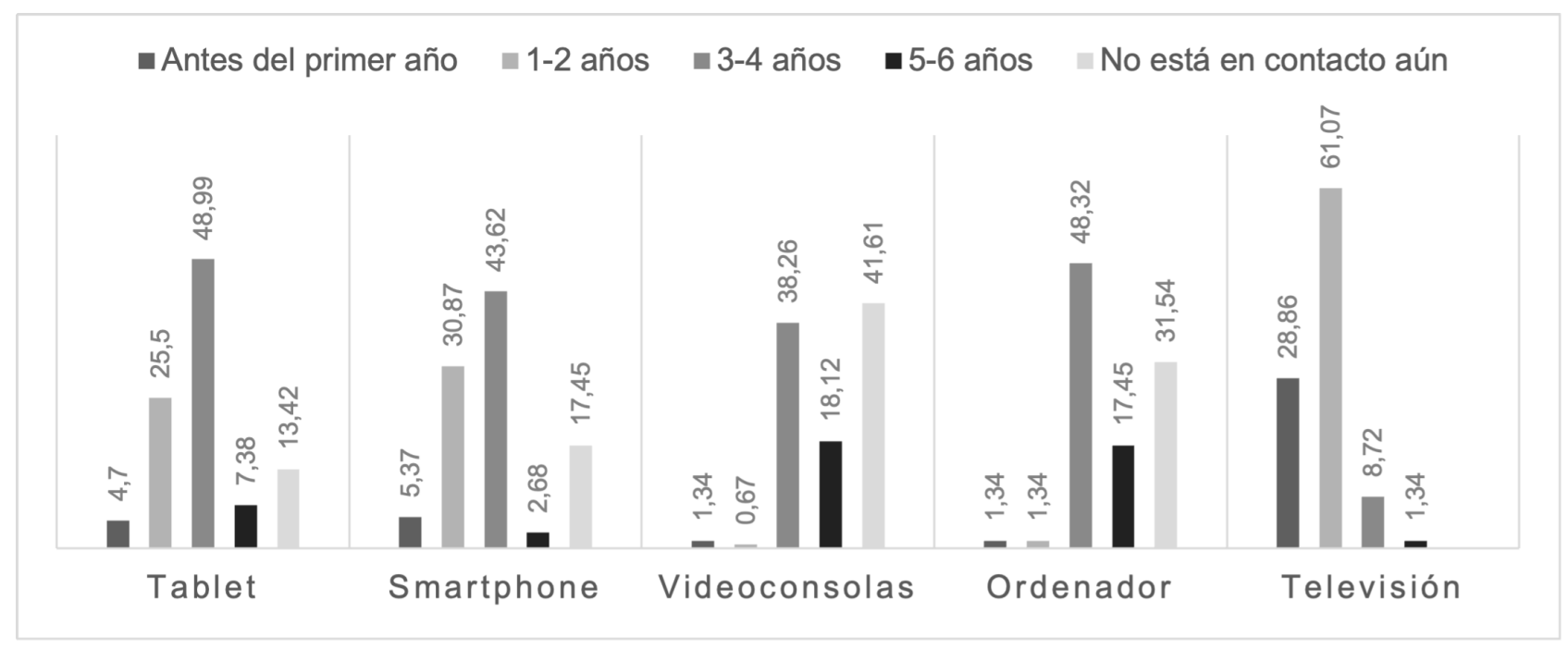

Figura 1. Gráfica sobre la edad de inicio en el uso de las TIC

Los datos anteriores representan el porcentaje de la muestra que entró en contacto con cada uno de los dispositivos dentro las TIC en las edades que se presentan. Si se busca el valor medio más bajo (es decir, el dispositivo con el que se entra en contacto antes) tomando como punto medio 3, es el ítem "Televisión" (M $=1.83$, d.t. $=.63)$ el que cumple con dicha premisa. Por el contrario, "Videoconsolas" $(\mathrm{M}=3.98$, d.t. $=.98)$ refleja el valor medio superior, mientras que los dos dispositivos móviles restantes -"Smartphone" ( $M=2.96$, d.t. $=1.12)$ y "Tablet" $(\mathrm{M}=2.99$, d.t. $=1.03)$ - toman valores muy similares.

Considerando los resultados, se ha creado una variable que agrupa las edades a las que se entra en contacto con cada uno de los dispositivos, denominándola "Edad a la que los menores entran en contacto con las TIC" (Tabla 7).

Tabla 7

Descriptivos de la variable "Edad a la que los menores entran en contacto con las TIC"

\begin{tabular}{|c|c|c|c|c|c|}
\hline que los & $\begin{array}{l}\mathbf{N} \\
149\end{array}$ & $\begin{array}{l}\text { Mínimo } \\
1.40\end{array}$ & $\begin{array}{l}\text { Máximo } \\
4.80\end{array}$ & $\begin{array}{l}\text { Media } \\
3.10\end{array}$ & $\begin{array}{l}\text { Desviación típica } \\
.64\end{array}$ \\
\hline
\end{tabular}

De esta forma, se observa que la puntuación media de la variable es superior al punto medio de la escala, 3 (escala de 1 a 5, siendo 1: Antes del primer año, 2: 1-2 años, 3: 3-4 años, 4: 5-6 años y 5: No está en contacto aún), situándose la edad media a la que entran en contacto con las TIC ligeramente por encima de los 3 años. 


\subsection{Dispositivos más utilizados por los menores dentro de las TIC}

Fijando la atención en los ítems relacionados con la frecuencia de uso de los diferentes dispositivos dentro de las TIC, se analizan los porcentajes reflejados en la Figura 2.

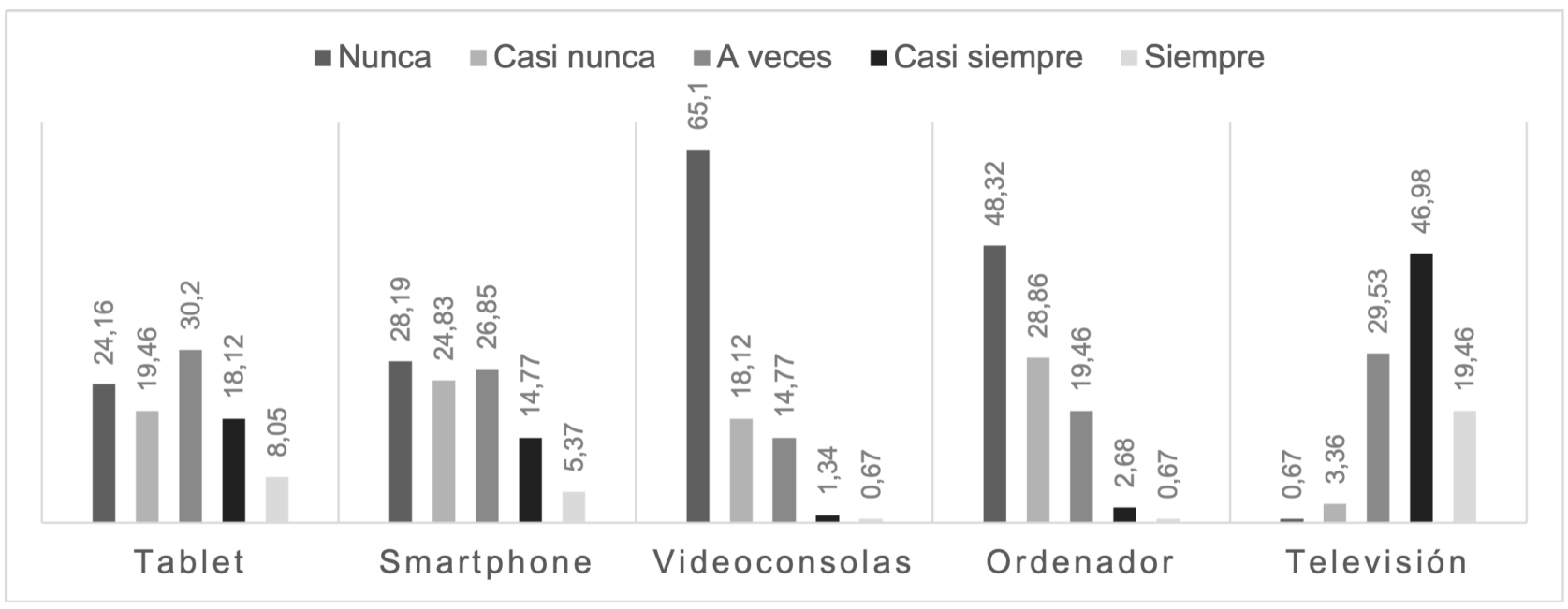

Figura 2. Gráfica sobre el uso de los diferentes dispositivos

De acuerdo con los resultados anteriores, se percibe que el único ítem que toma un valor medio superior al punto medio de la escala es "Televisión" $(\mathrm{M}=3.81$, d.t. $=.81)$, lo cual indica que se trata del dispositivo empleado con mayor frecuencia. En lo que respecta a los dispositivos móviles, "Videoconsolas" $(\mathrm{M}=1.54$, d.t. = .84) representa el valor medio más bajo, mientras que "Tablet" $(\mathrm{M}=2.66$, d.t. $=1.25)$ y "Smartphone" $(\mathrm{M}=2.44$, d.t. $=1.20)$ muestran valores superiores -nuevamente muy parejos-, situándose los tres casos por debajo del punto medio de la escala (3).

\subsection{Finalidad de uso de las TIC por parte de los menores}

Atendiendo a los ítems referidos a la finalidad de uso de las TIC, los datos obtenidos quedan representados en la Figura 3.

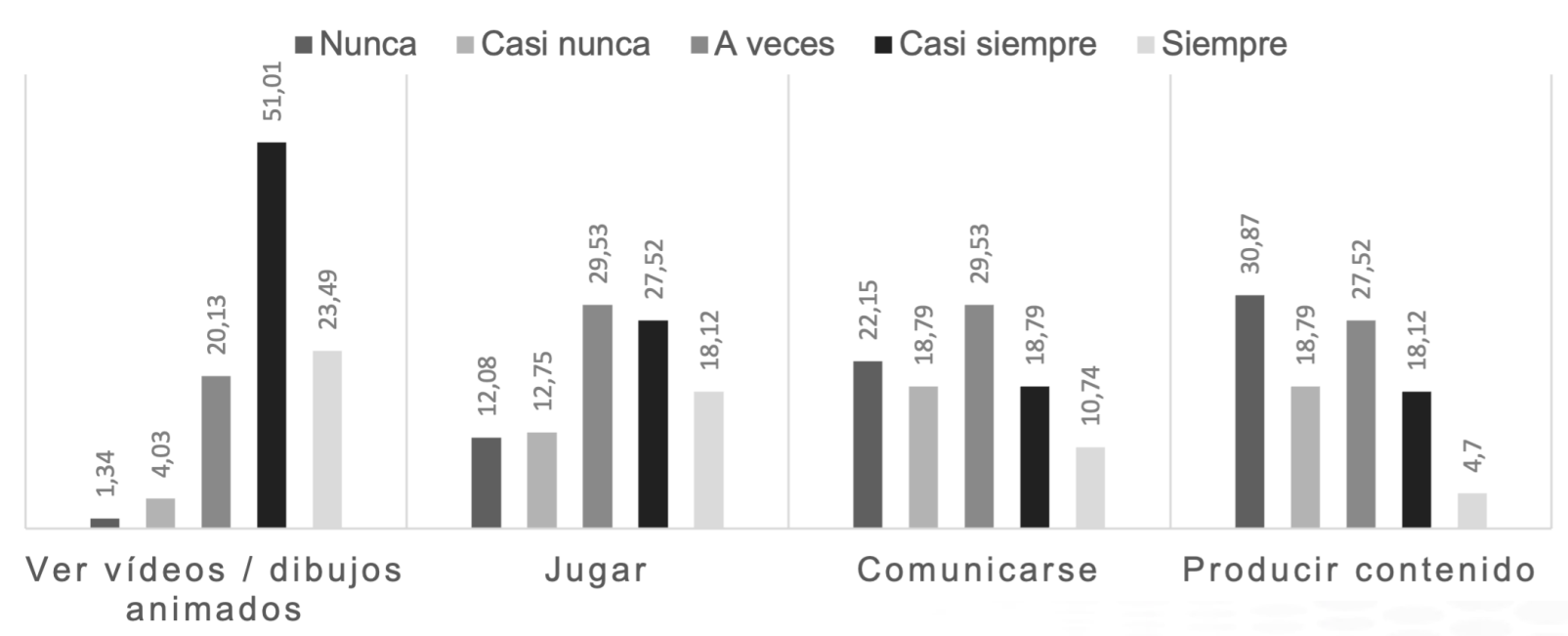

Figura 3. Gráfica sobre la finalidad de uso de las TIC por parte de los menores 
Considerando la gráfica anterior y comparando los resultados, se advierte una disminución gradual en el valor medio de los cuatro ítems que se proponen, la cual abarca un rango que oscila desde $(M=3.91$, d.t. $=.85)$, correspondiente a "Ver vídeos / dibujos animados", hasta $(M=2.47$, d.t. $=1.23)$, relativo a "Producir contenido". De este modo, dos de las puntuaciones ("Ver vídeos / dibujos animados" y "Jugar") se encuentran por encima del punto medio de la escala (3), mientras que las restantes ("Comunicarse" y "Producir contenido") se localizan por debajo de este.

\subsection{Intención con la que las familias promueven el uso de las TIC}

Finalmente, se analizan los ítems relativos a la intención con la que las familias exponen a los más pequeños a las TIC. Los resultados extraídos se muestran en la Figura 4.

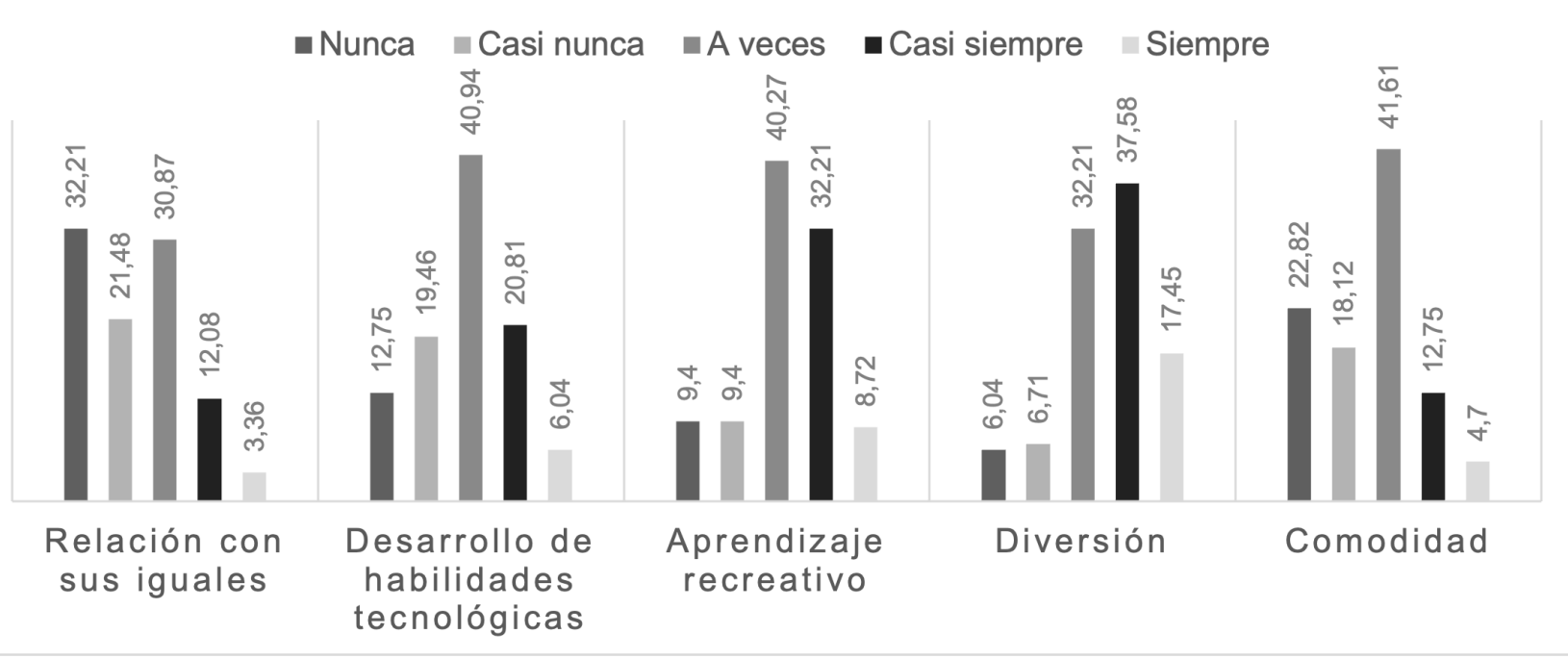

Figura 4. Gráfica sobre la intención con la que las familias promueven el uso de las TIC

Atendiendo a los resultados, se advierte cómo únicamente dos de los valores medios son superiores al punto medio de la escala (3). Así, la intención de uso con mayor puntuación es "Diversión" ( $\mathrm{M}=3.54$, d.t. $=1.05)$, seguida por "Aprendizaje recreativo" $(M=3.21$, d.t. $=1.05)$. En contraposición, las puntuaciones más bajas se encuentran en "Comodidad" y en "Relación con sus iguales", con valores de $(\mathrm{M}=2.58$, d.t. $=1.12)$ y de (M $=2.33$, d.t. $=1.15$ ) respectivamente.

\section{Discusión}

Tras analizar el impacto de las TIC en el ocio de los menores de 3-6 años, se observa que el tiempo de uso de las mismas oscila -por lo general- alrededor de 1 hora diaria. Sin embargo, esta cifra aumenta durante los fines de semana, ya sea en solitario o en compañía de los progenitores. Además, se constata que el tiempo dedicado al empleo de las TIC crece a medida que lo hace la edad de los niños, lo cual se relaciona con los resultados obtenidos en varias investigaciones previas (Fernández, 2017; INE, 2019; Labrador et al., 2015).

Continuando en la línea de lo anterior, se analiza la edad a la que los menores entran en contacto con las mismas, advirtiendo cómo esta se sitúa ligeramente por encima de los 3 años. Este resultado diverge mínimamente respecto a los datos obtenidos por algunos autores, quienes sitúan este momento previo a dicha edad (Cánovas et al., 2014; Chaudron et al., 2018; Roca, 2015; Sivrikova et al., 2020). No obstante, esto último sí que ocurre en el caso de la televisión, a la cual el $89.93 \%$ de los progenitores exponen a sus hijos antes de este momento. De igual forma, también se observa -de acuerdo con el informe de GAD3 (2018) y con las investigaciones de Chaudron et al. (2018) y de Feijóo (2015)- que este es el dispositivo utilizado con mayor frecuencia por parte de los más pequeños, contrastando con varios autores que sitúan a dos de los dispositivos móviles -tablet y smartphone- en este lugar (Chaudron et al., 2018; Garmendia et al., 2016; INTEF, 2016; Fernández, 2017; Martínez et al., 2019; Pérez, 2018).

Por su parte, se ha podido comprobar que la mayoría de los padres proponen a los más pequeños el uso de las TIC con la intención principal de que se diviertan y aprendan de manera recreativa, constituyendo así los usos 
predominantes de las mismas. Estos resultados divergen respecto a algunas investigaciones previas (Cánovas et al., 2014; Chaudron et al., 2018) que apuntan a la comodidad como el propósito fundamental que guía a las familias en la elección de las propuestas. Sí coinciden, sin embargo, varios estudios anteriores (Cánovas et al., 2014; Carrillo, 2015; Chaudron et al., 2018; INTEF, 2016) con los resultados que revela esta investigación respecto a la intención con la que los menores utilizan las TIC, indicando que el juego y la visualización de dibujos animados son los usos más habituales entre ellos.

\section{Conclusiones}

Una vez discutidos los resultados obtenidos en la presente investigación a la luz de la revisión teórica previa, y constatando el plano principal que toman las actividades que recurren al soporte tecnológico dentro del ocio de los menores, en la Tabla 8 se muestran algunas propuestas de gestión de uso de las TIC destinadas a las familias que complementan y se añaden a las pautas aportadas anteriormente por la investigación de Ramírez-García et al. (2020).

\section{Tabla 8}

Propuestas de gestión del uso de las TIC para las familias

1. Ofrecer un buen modelo de uso digital, mostrando cómo y para qué pueden ser utilizadas correctamente las TIC: hacerles competentes tecnológicamente.

2. Fijar un horario concreto y responsable de uso de las TIC, atendiendo a la edad de los niños.

3. Conocer a qué contenidos y actividades acceden los menores:

- En qué consisten.

- Qué valores transmiten.

- Qué información personal solicitan.

4. Ralentizar el ritmo de vida de los más pequeños, huyendo de la inmediatez -e incluso anticipación- de respuesta a sus demandas que ofrecen las TIC.

5. Proponer alternativas de ocio, como por ejemplo:

- CON soporte tecnológico:

- Juegos educativos estandarizados, con la consiguiente regulación horaria.

- SIN soporte tecnológico:

- Actividad física, considerando la carga adecuada en función de la edad.

- Actividades culturales (literatura, teatro, música, etcétera).

- Actividades en relación con el entorno.

- Juego simbólico.

Finalmente, como principal limitación a considerar en esta investigación se toman las características de la muestra que, al acotarse a una única localidad, no permiten extrapolar ni generalizar los resultados obtenidos. En este sentido, como futuras líneas de investigación resultaría muy interesante diversificar la muestra, ampliando el rango de edad de los menores, así como introducir nuevas variables como la influencia del contexto urbano o rural en los resultados obtenidos.

\section{Referencias}

Albert, M. J. (2007). Aspectos fundamentales sobre investigación (E. M. Albert, Ed.). La: McGraw-Hill.

Álvarez, E., y Alejaldre, L. (2019). Las TIC como mediadoras en el aprendizaje de lenguas extranjeras en Educación Infantil. Aproximación desde el enfoque natural. Tejuelo(30), 175-206.

Alvarez-Pitti, J., Casajús-Mallén, J. A., Leis-Trabazo, R., Lucía, A., de Lara, D. L., Moreno-Aznar, L. A., y Rodríguez-Martínez, G. (2020). Ejercicio físico como «medicina» en enfermedades crónicas durante la infancia y la adolescencia. Anales de Pediatría, 92(3), 173.e1-173.e8. Retrieved from https://dx.doi.org/10.1016/ j.anpedi.2020.01.010 10.1016/j.anpedi.2020.01.010

Cánovas, G., García, A., Oliaga, A., y Aboy, I. (2014). Menores de Edad y Conectividad Móvil en España: Tablets y Smartphones. Centro de Seguridad en Internet para los Menores en España: Protégeles. Retrieved from https://bit.ly/339oVtf

Carrillo, S. C. (2015). Infancia y Socialización Mediática: el papel de las Tecnologías de la Información y la Comunicación (TIC) en la socialización de un grupo de niños y niñas de 5 y 6 años de edad. Retrieved from https://bit.ly/30gF26g

Cepal, N., y Unicef. (2016). El derecho al tiempo libre en la infancia y adolescencia. Desafíos: Boletín de la infancia y adolescencia, 19, 1-12. Retrieved from https://bit.ly/3hTa4qR 
Chaudron, S., Gioia, R. D., y Gemo, M. (2018). Young Children (0-8) and Digital Technology. A qualitative study across Europe. European Union. Retrieved from http://doi.org/10.2760/294383\%20

Cruz, M. A., Pozo, M. A., Aushay, H. R., y Arias, A. D. (2019). Las Tecnologías de la Información y de la Comunicación (TIC) como forma investigativa disciplinaria con un enfoque intercultural para el proceso de formación estudiantil. e-Ciencias de la Información, 9(1), 2-15. Retrieved from https://doi.org/10.15517/ eci.v1i1.33052

de Estadística., I. N. (Ed.). (2019). Encuesta sobre Equipamiento y Uso de Tecnologías de Información y Comunicación en los Hogares. Retrieved from https:/ /bit.ly/2Pbdd9p

de la Rosa, O. M. A., y Angulo, L. M. V. (2019). Análisis factorial de las actitudes de niños de 6 a 16 años con dispositivos auditivos hacia la educación inclusiva. Aula Abierta, 48(2), 129-129. Retrieved from https://dx.doi.org/10.17811/rifie.48.2.2019.129-138 10.17811/rifie.48.2.2019.129-138

de-la Salud, O.-M. (2019). Guidelines on physical activity, sedentary behaviour and sleep for children under 5 years of age. Retrieved from https://bit.ly/312eB3y

de Pediatría., A. A. (Ed.). (2016). Media Use in School-Aged Children and Adolescents. Pediatrics, 138(5), 1-6. de Tecnologías Educativas y de Formación del Profesorado, I. N. (Ed.). (2016). Uso de las tecnologías por niños de hasta ocho años. INTEF. Retrieved from https://bit.ly/3jYkF5L

Díaz, R., y Aladro, M. (2016). La relación entre uso de las nuevas tecnologías y sobrepeso infantil, como problema de salud pública. RqR Enfermería Comunitaria, 4(1), 46-51.

Espinoza-Santacruz, F. J., y Flores-Urgiles, C. H. (2019). Aplicación de tecnologías de la información en el desarrollo del lenguaje de niños con dificultades de comunicación. Polo del Conocimiento: Revista Científico Profesional, 4(5), 116-116. Retrieved from https://dx.doi.org/10.23857/pc.v4i5.968 10.23857/pc.v4i5.968

Feijóo, B. (2015). La infancia ante las pantallas: análisis del consumo de medios audiovisuales (TV, videojuegos e internet) entre los niños gallegos del último curso de primaria en 2010. Retrieved from https://bit.ly/362fuwX Fernández, A. (2017). Las nuevas tecnologías en la primera infancia. Retrieved from https://bit.ly/3hXZU8j GAD3. (2018). Retrieved from https://bit.ly/3hT85CV

Garmendia, M., Jiménez, E., Casado, M. A., y Mascheroni, G. (2010). Net Children Go Mobile: Riesgos y oportunidades en internet y el uso de dispositivos móviles entre menores españoles. Red.es/Universidad del País Vasco/Euskal Herriko Unibertsitatea.

Gjelaj, M., Buza, K., Shatri, K., y Zabeli, N. (2020). Digital Technologies in Early Childhood: Attitudes and Practices of Parents and Teachers in Kosovo. International Journal of Instruction, 13(1), 165-184.

Hernández, R., Fernández, C., y Baptista, P. (2006). Similitudes y diferencias entre los enfoques cuantitativo y cualitativo. Metodología de la Investigación, 1-30.

Hernández, R., Fernández, C., y Baptista, P. (2007). Definición del alcance de la investigación a realizar: Exploratoria, descriptiva, correlacional o explicativa. En R. Hernández, C. Fernández \& P. Baptista, Fundamentos de metodología de la investigación, 57-72.

Labrador, F., Requesens, A., y Helguera, M. (2015). Guía para padres y educadores sobre el uso seguro de Internet, móviles y videojuegos. Fundación Gaudium. Retrieved from https://bit.ly/2Xi2yy6

Las nuevas tecnologías en niños y adolescentes. Guía para educar saludablemente en una sociedad digital. (2015). In G. Roca (Ed.), . Hospital Sant Joan de Déu. Retrieved from https://bit.ly/3fg9Bxe

L'ecuyer, C. (2017). Educar en el asombro. Plataforma Editorial.

León-Llorente, C. (2020). Robotización, ¿solo cambiará el empleo? Revista empresa y humanismo, 23(1), 9-33. Retrieved from https://doi.org/10.15581/015.xxiii.1.9-33

López-Castro, L., Núñez, J., y Cambeiro, M. C. (2019). Medición parental del uso de Internet: Una estrategia educativa para minimizar los riesgos de la infancia. In G. d'Investigació i Formació Educativa i Social (Ed.), Congreso Internacional SIPS: "Pedagogía social, investigación y familias" (p. 59-68). Retrieved from https:// bit.ly/3kHytkJ

López-Cirugeda, I., y López-Campillo, R. M. (2016). El método sistemático-sintético de lectoescritura Phonics como herramienta para la adquisición de la fonética inglesa. Revista Fuentes, 18(2), 183-195.

Martínez-Pastor, E., Catalina-García, B., y de Ayala-López, M.-C. L. (2019). Smartphone, menores y vulnerabilidades. Revisión de la literatura. Revista Mediterránea de Comunicación, 10(2), 257-268. Retrieved from https://dx.doi.org/10.14198/medcom2019.10.2.5 10.14198/medcom2019.10.2.5

Muñoz-Borja, P., García-Ruiz, R., y Aguaded, I. (2020). La educomunicación en la documentación pública, privada y académica colombiana en su compresión de la cuestión social. Revista Fuentes, 1(22), 1-12. Retrieved from https://dx.doi.org/10.12795/revistafuentes.2020.v22.i1.01 10.12795/revistafuentes.2020.v22.i1.01 Paniagua, H. (2018). El impacto de las pantallas: televisión, ordenador y videojuegos. Pediatría Integral, 22(4), 178-186.

Pérez, A. (2018). Uso de smartphones y redes sociales en alumnos/as de Educación Primaria. Prisma social, 20, 76-91. Retrieved from https://bit.ly/2G1LYN0

Prensky, M. (2001). Digital Natives, Digital Immigrants. On the Horizon, 9(5), 1-6. Retrieved from https://bit.ly/ 2RSClxm 
Quiroga, L. P., Vanegas, O. L., y Pardo, S. (2019). Ventajas y desventajas de las tic en la educación "Desde la primera infancia hasta la educación superior". Revista de educación y pensamiento, 26, 77-85. Retrieved from https://bit.ly/33TT1zK

Ramírez-García, A., Salcines-Talledo, I., y González-Fernández, N. (2020). Los dispositivos móviles en el hogar. Interés formativo de las familias españolas. REOP - Revista Española de Orientación y Psicopedagogía, 31(1), 43-43. Retrieved from https://dx.doi.org/10.5944/reop.vol.31.num.1.2020.27286 10.5944/reop.vol.31.num .1 .2020 .27286

Rojo, T., y Dudu, S. (2017). Los "juegos serios" como instrumentos de empoderamiento y aprendizaje sociolaboral inclusivo. Revista Fuentes, 19(2), 95-109.

Sáez, J. M. (2017). Investigación educativa. Fundamentos teóricos, procesos y elementos prácticos. UNED. Serna-Rodrigo, R. (2020). Posibilidades de los videojuegos en el ámbito de la Didáctica de la Lengua y la Literatura. EDMETIC. Revista de Educación Mediática y TIC, 9(1), 104-125.

Sivrikova, N. V., Ptashko, T. G., Perebeynos, A. E., Chernikova, E. G., Gilyazeva, N. V., y Vasilyeva, V. S. (2020). Parental reports on digital devices use in infancy and early childhood. Education and Information Technologies, 25, 3957-3973. Retrieved from https://dx.doi.org/10.1007/s10639-020-10145-z 10.1007/s10639-020-10145-z

Soto, A., De-Miguel, N., y Pérez, V. (2018). Abordaje de adicciones a nuevas tecnologías: Una propuesta de prevención en contexto escolar y tratamiento de rehabilitación. Papeles del Psicólogo, 39(2), 120-126. Retrieved from https://doi.org/10.23923/pap.psicol2018.2867

Takacs, Z. K., Swart, E. K., y Bus, A. G. (2015). Benefits and Pitfalls of Multimedia and Interactive Features in Technology-Enhanced Storybooks. Review of Educational Research, 85(4), 698-739. Retrieved from https:// dx.doi.org/10.3102/0034654314566989 10.3102/0034654314566989

Turk, V. (2018). Entendiendo a la Generación Alfa. Hotwire, The Global Communications Agency. Retrieved from https://bit.ly/3ggfwE3

Unicef. (2014). Acompañando a los nativos digitales. Retrieved from https://bit.ly/3164UB6

UNICEF. (2017). Niños en un mundo digital. El estado mundial de la infancia 2017. Retrieved from https:// doi.org/10.18356/fb1d52cf-es

UNICEF. (2018). Niños, niñas y adolescentes conectados: Informe Kids Online Uruguay. Retrieved from https://bit.ly/2PILrXv 